\title{
Erratum: Measurement of zinc stable isotope ratios in biogeochemical matrices by double-spike MC-ICPMS and determination of the isotope ratio pool available for plants from soil
}

Tim Arnold • Maria Schönbächler • Mark Rehkämper • Schuofei Dong • Fang-Jie Zhao • Guy J. D. Kirk •

Barry J. Coles • Dominik J. Weiss

Published online: 27 November 2010

(C) Springer-Verlag 2010

Erratum to: Anal Bioanal Chem

DOI 10.1007/s00216-010-4231-5

Regrettably, there was a mistake in Equation 4 of the original article.

The correct form of Equation 4 is

$\delta^{66} \mathrm{Zn}_{\text {sample(true })}=\delta^{66} \mathrm{Zn}_{\text {sample(measured })}-\delta^{66} \mathrm{Zn}_{\text {London(measured })}$

The online version of the original article can be found at http://dx.doi. org/10.1007/s00216-010-4231-5.

T. Arnold $(\bowtie) \cdot$ M. Schönbächler $\cdot$ M. Rehkämper $\cdot$ S. Dong $\cdot$

B. J. Coles $\cdot$ D. J. Weiss

Department of Earth Science and Engineering,

Imperial College London,

London SW7 2AZ, UK

e-mail: tarnold@ucsd.edu

T. Arnold

Scripps Institution of Oceanography,

University of California, San Diego,

La Jolla, CA 92093, USA

M. Schönbächler

School of Earth, Atmospheric, and Environmental Science,

The University of Manchester,

Manchester M13 9PL, UK

F.-J. Zhao

Rothamsted Research,

Harpenden, Herts AL5 2JQ, UK

G. J. D. Kirk

National Soil Resources Institute, Cranfield University,

Cranfield MK3 0AL, UK

D. J. Weiss

The Natural History Museum,

London SW7 5PD, UK 